\title{
High Dose Statins: Is it Beneficial over Conventional Dose of Statin in Acute Coronary Syndrome? \\ AEM M Islam ${ }^{1}$, AAS Mazumder ${ }^{2}$ \\ ${ }^{1}$ Dept. of Cardiology, Dhaka Medical College, Dhaka, ${ }^{2}$ Dept. of Cardiology, NICVD, Dhaka
}

Keywords-

Statin, Acute coronary

syndrome.

\begin{abstract}
:
Increasing burden of cardiovascular disease risk factors has led to increase in the incidence in the coronary artery disease (CAD). Advancements in medical and surgical care of patients with heart disease have led to a large number of people surviving acute coronary events. Patients with acute coronary syndrome (ACS) are at high risk for recurrent coronary events, sudden death, and all cause mortality. Conventional revascularization therapies reduce the risk of further ischaemia but do not affect the underlying atherosclerotic disease. Statins have a proven record in the secondary prevention of coronary heart disease. Furthermore, statins have been shown to exert various pleotropic effects, which may stabilize vulnerable atherosclerotic plaques. Therefore, initiating, statin therapy in patients with established CAD is both beneficial and more cost effective. Trials conducted in recent times have addressed the issue of whether higher dose statin therapy provides further reductions in the risks of cardiovascular events following an acute coronary syndrome diagnosis, over and above that provided by a conventional dose statin regimen. However, recent trials of high dose statin therapy in patients with acute coronary syndromes have yielded conflicting results. The aim of this article is to provide a critical and up-to-date summary of the findings of clinical trials of intensive statin therapy, including a brief overview of safety and efficacy of such treatments.
\end{abstract}

(Cardiovasc. j. 2010; 2(2) : 245-251)

\section{Introduction:}

Globally, the increasing burden of vascular disease risk factors has led to increase in the incidence of coronary artery disease. Dyslipidaemia constitute a major risk factor for atherosclerosis and coronary artery disease, and their proper recognition and management can reduce cardiovascular and total mortality rates. ${ }^{1}$ Statins inhibit HMG-CoA reductase and prevent the formation of mevalonate, the rate limiting step of sterol synthesis. To maintain cellular cholesterol homeostasis, expression of the LDL-R increases and the rate of cholesteryl ester formation declines. These homeostatic adjustments to HMG-CoA reductase inhibition increase LDL cholesterol clearance from plasma and decrease hepatic production of VLDL and LDL. ${ }^{1}$ Atherosclerosis is an inflammatory disease. Statins decrease C reactive protein, ${ }^{2}$ induce apoptosis in smooth muscle cells, alter collagen content of atherosclerotic plaques alter endothelial function, decrease the inflammatory component of plaques. ${ }^{3,4}$
Acute Coronary syndrome(ACS) refers to the spectrum of acute myocardial ischaemia, including Unstable angina(UA), Non ST segment elevation myocardial infarction (NSTEMI) and ST segment elevation myocardial infarction. ${ }^{5-7}$ The risk of recurrent coronary events is greater in the weeks and months following ACS. Despite the widespread use of conventional therapies aimed at modifying platelet function and coagulation cascade to reduce the risk of further ischaemia, patients who gave experienced an ACS continue to at higher cardiac risk. Nearly $25 \%$ of men and $38 \%$ of women die within 1 year of a first myocardial infarction (MI). ${ }^{8}$. These statistics highlight the need for improve strategies that

target the Pathophysiologic mechanism operating in ACS and treat the underlying atherosclerotic disease. Evidence from numerous large, randomized trials unequivocally demonstrates the ability of statins to reduce coronary morbidity \& mortality. ${ }^{9}$ Though the benefits of statin therapy in patients with stable coronary artery disease are

Address of Correspondence: Dr. A E M Mazharul Islam, Department of Cardiology, Dhaka Medical College, Dhaka, Bangladesh. 
clearly recognized, it is only recently the positive impact of initiating statin treatment immediately following the occurrence of ACS has emerged. ${ }^{10}$

Atherosclerosis and its progression to ACS: Atherothombosis is the major cause of ACS. It is now clear that atherosclerosis is not an inevitable consequence of aging but rather chronic inflammatory process that can be converted to an acute clinical event by plaque rupture and arterial thrombosis. ${ }^{11}$ Contemporary models suggest that atherosclerosis plaques are not merely passive structures but dynamic inflammatory lesion. Fatty streaks, which can be detected in the arterial intima as early as adolescence, progress into more complicated raised lesions composed of inflammatory cellular material, along with cholesterol esters. Lesions develop into mature atheroma containing fibrous tissue elements, inflammatory elements, and extracellular deposits of cholesterol and are vulnerable to rupture. ${ }^{12,13}$ Although the exact inciting factor factors of the vulnerable plaque rupture are not fully understood, inflammation is accepted to be the pivotal event. ${ }^{14}$ The rupture of an unstable atherosclerotic plaque results in the lipid core coming into contact with circulating blood, which triggers platelets activation and coagulation, leading to thrombosis. ${ }^{15}$

\section{Therapeutic Strategies in ACS and Role of lipid} lowering:

While the underlying molecular processes of atherogenesis are complex, they are amenable to therapeutic intervention. After an ACS, the primary goal of therapy is to reduce the risk of further ischaemic complication. Antiplatelet therapy is the foundation of immediate ACS management. Therapies to reduce platelet aggregation include aspirin, the thienopyridine agent Clopidogrel, and platelet IIb/IIIa antagonists. In addition, a fundamental aspect of treatment involves the use of anticoagulant agents such as unfractionated heparin and newer low molecular weight heparin, Thrombolytic agents are used in early stages of ST elevated myocardial infarction. Anti-ischaemic treatments include the use of nitrates and $B$ blockers. Neurohumoral agents such as ACE inhibitors, $B$ blockers, and aldosterone antagonists are utilize for early and long term cardioprotection. ${ }^{16}$
While the benefit of statin therapy in patients with stable coronary artery disease is clearly recognized, the positive impact of the initiation of statin therapy immediately following occurrence of ACS has emerged only recently. Both STEMI and UA/ NSTEMI frequently require intensive care treatment and these patients are at high risk for recurrent coronary events, sudden cardiac death and all cause mortality. The stabilization of vulnerable plaque is a critical aspect in preventing these events following ACS. Despite significant advances in Antiplatelet and Antithombotic therapy, these therapeutic options alone do not appear to suffice in stabilizing unstable plaque. Through their pleotropic affect statins are viewed as important contributions to plaque stabilization.

A critical aspect of preventing future coronary event is stabilizing vulnerable lesions. Although significant therapeutic advances in the treatment of ACS have been made with Antiplatelet and Antithombotic therapy during the past decade, these therapies alone do not appear to completely stabilize unstable plaques. ${ }^{17}$ Results from several clinical trials suggest that early administration of high dose potent statin therapy following ACS may stabilize vulnerable plaques decreasing adverse outcomes in both the short \& long term. ${ }^{6}$ The likely mechanism of benefit are not solely due to the lipid lowering effects of statins but, at least in part, to the variety of anti-inflammatory and antiproliferative effects, commonly described as pleotropic effects exerted by statins. ${ }^{18}$ Pleotropic effects commonly ascribed to statins include improvements in endothelial function and vasomotion, reduction in platelet aggregability and thrombus formation, fibrinolytic and antioxidant activity as well as decrease in matrix degradation due to reduction in macrophage metalloproteinase production and increase in collagen content. Furthermore, statins are proposed to reduce inflammation within plaques. ${ }^{17}$ The most widely examined inflammatory biomarker is high sensitivity C-Reactive protein (hsCRP). Elevated levels of hsCRP have been correlated with increased cardiovascular risk and morbidity. ${ }^{19} \mathrm{C}$ reactive protein bind to oxidized low density lipoprotein cholesterol (LDL-C) and apoptotic cells but not to native LDL-C or healthy cells, signifying an association with atherosclerotic plaques. Reduction in hsCRP levels achieved with intensive 
statin therapy appear to correlate with increased clinical benefit in patients who have experienced an ACS. $^{20}$

Rationale for use of statin in ACS-

1. Stabilization of vulnerable plaque

a. Improvement in endothelial function

b. Increase in bioavailability

c. Anti-oxidant properties

2. Decrease MACE in patients undergoing PCI

3. Decrease mortality in ACS patients

4. Antiarrythmic action

5. In hospital administration improves compliance

\section{Clinical Trials with Statins in ACS:}

A number of retrospective and observational studies have suggested statin therapy immediately after an ACS is associated with significantly reduced rates of recurrent coronary events and death. ${ }^{21}$ these have been followed up by large-scale, randomized, controlled trials.

\section{MIRACAL Study:}

The Myocardial Ischaemia Reduction with Aggressive Cholesterol Lowering (MIRACAL) ${ }^{22}$ trial was the first to demonstrate a reduced rate of recurrent cardiac events by statin therapy. In this study 8036 patients with unstable angina and non $\mathrm{Q}$ infarction were randomized within 24-96 h after hospital admission to receive either atorvastatin $80 \mathrm{mg}$ or placebo in addition to state of the art therapy for 4 months after ACS. The primary end point of the trial- death, cardiac arrest, myocardial infarction or worsening unstable angina requiring emergency hospitalization at 16 weeks - showed a relative risk reduction of $16 \%$ [ $95 \%$ confidence interval (CI), 0-30; P-0.048; absolute risk reduction, $2.6 \%$ ]

\section{PROVE IT-TIMI 22 Study:}

The Pravastatin or Atorvastatin Evaluation and Infection Therapy - Thombolysis in Myocardial Infarction 22( PROVE IT-TIMI 22) ${ }^{23}$ - has extended the findings of the MIRACAL trial, while also demonstrating the greater benefit of intensive lipid lowering therapy with atorvastatin $80 \mathrm{mg}$, compared to conventional lipid lowering therapy with pravastatin $40 \mathrm{mg}$. This trial randomized 4162 patients hospitalized for an ACS (NSTEMI and STEMI). Patients were included within 10 days of their index event, and were randomized to either pravastatin $40 \mathrm{mg}$ daily or atorvastatin $80 \mathrm{mg}$ daily. Of note this study also included a significant number of post percutaneous revascularization patients (69\%). The results showed a strong trend toward benefit in the primary end point (death from any cause or a major cardiovascular event) in high dose atorvastatin group within 30days. A statistically significant decrease in the primary endpoint arose at 180 days (relative risk reduction of $16 \%, \mathrm{p}$ value $=0.005$; absolute risk reduction, $3.9 \%$ ). The secondary end points (revascularization, unstable angina requiring hospitalization, and the combined end point of MI, revascularization, or death from coronary heart disease) also showed a significant decrease during the overall 2 year assessment period. The benefit derived from intensive versus conventional lipid lowering therapy arouse on top of background evidence based ACS therapy (including antiplatelet therapy $B$ blocker and ACE inhibitors in a large majority patients).

The Aggrastat to Zocor(A to Z) Trial:

The Aggrastat to Zocor (A to Z) trial ${ }^{24}$ a large randomized, double-blind, controlled trial, compared the earlty initiation of an intensive statin regimen with delayed initiation of a less intensive regimen in patients with ACS. The investigators randomized 4497 patients following an ACS event to receive either simvastatin $80 \mathrm{mg} / \mathrm{d}$ for 1 month and then $80 \mathrm{mg} / \mathrm{d}$ thereafter $(\mathrm{n}=2265)$ or to regimen of placebo for 4 month and then simvastatin 20 $m g$ thereafter $(n=2232)$. The intensive regimen failed to show a statistical significant benefit for reducing the composite end point of cardiovascular death, MI, readmission for ACS, or stroke compared with less intensive regimen(absolute risk reduction, $2.3 \%$; hazard ratio $0.89 ; 95 \% \mathrm{CI}, 0.76$ to 1.04; $\mathrm{p}=0.14)$. A statin trial failing to achieve a statistical significance was a major set back. But when this trial was critically analyzed it was found 
that the CRP level reductions was only $17 \%$ in this trial as compared to $34 \%$ and $38 \%$ in the MIRACAl and PROVE- IT trial, More than the lipid level, it is the anti- inflammatory action of statins which is more important in ACS. Secondly the benefit was not seen in the first three months in the trial but seen thereafter. This could be due to the fact that statin was titrated its maximum dose only after 3 month.

Based on the finding from these three large randomized trials, it has been speculated that early benefits of statin therapy may be caused largely by anti-inflammatory effects, whereas late benefits are more likely to be lipid modulated.

\section{SEARCH Trial:}

Study of the Effectiveness of Additional Reductions in Cholesterol and Homocystein SEARCH Trial ${ }^{25}$ is the largest randomized trial to assess directly the efficacy and safety of lowering LDL cholesterol more than is typically done. It involved 12,000 men and women who had survived a heart attack. 6,000 were given a higher dose of $80 \mathrm{mg}$ simvastatin daily, and 6,000 were given a more standard dose of $20 \mathrm{mg}$ simvastatin daily. During an average of 6.7 years of treatment, the $80 \mathrm{mg}$ simvastatin regimen lowered $\mathrm{LDL}$ cholesterol by an average of $14 \mathrm{mg} / \mathrm{dl}(0.35 \mathrm{mmol} /$ l) more than the $20 \mathrm{mg}$ simvastatin regimen. This additional reduction in LDL cholesterol was associated with $6 \%$ fewer heart attacks, strokes or revascularizations. Although not statistically significant on its own, the risk reduction in SEARCH is entirely consistent with the benefit observed in previous statin trials. When it was combined in a meta analysis with the 4 previous trials that had directly compared more versus less intensive statin regimens, lowering $\mathrm{LDL}$ cholesterol further by an average of $20 \mathrm{mg} / \mathrm{dl}$ was shown to produce highly significant $15 \%$ further reductions in heart attacks, strokes and revascularisation procedures.

In hospital administration of statins improves long term compliance:

It has also been seen that in hospital administration of statins improves long term compliance. Patients will be more likely to understand the importance of lipid lowering therapy if statin is started during hospitalization for ACS and then he is less likely to discontinue the therapy. The Cardiac Hospitalization Atherosclerosis management Programme (CHAMP) addressed this issue in the pre discharged cardiac patients. ${ }^{26}$ They found that the 1 year compliance rate increased from $10 \%$ to $90 \%$ when statins were prescribed during hospitalization for ACS than when it is prescribed on out patient basis.

Statins and percutaneous coronary intervention in ACS:

The pleotropic effects of statins are also being studied for their effect following PCI in Acute coronary syndrome. The Atorvastatin for reduction of Myocardial damage During Angioplasty- Acute Coronary Syndromes (ARMYDA-ACS) trial demonstrated that short term pretreatment with atorvastatin $80 \mathrm{mg}$ reduces the incidences of cardiac events in ACS patients undergoing early PCI, a benefit largely driven by significant reduction in post procedural MI. ${ }^{27}$ This trial randomized 171 patients with Non ST segment elevation ACS to pretreatment with atorvastatin ( $80 \mathrm{mg} 12$ hour before, and 40 $\mathrm{mg}$ immediately prior to PCI) or to placebo. All patients were given $600 \mathrm{mg}$ loading dose of Clopidogrel and long term atorvastatin treatment (40 mg/day). The primary end point, a composite of death, MI, and TVR at 30 days was significantly lower among ACS patients pretreated with atorvastatin compared with those treated with placebo ( $5 \%$ vs. $17 \%$ respectably, $p=.01$ ). The incidence of MACE was primarily driven by significant differences in post procedural MI, with reductions in Ck-MB and troponin levels in patients pretreated with atorvastatin. In multivariate analysis, pretreatment with atorvastatin was associated with an $88 \%$ reduction in relative risk of MACE at 30 days. If confirmed by larger randomized trials, this study may support the indication for "upstream" administration of high dose statins in patients with ACS undergoing an early invasive strategy. 
Table-I

Randomized clinical trials of statins in critical care

\begin{tabular}{|c|c|c|c|c|c|c|}
\hline Sl. No. & Trial & $\mathrm{n}$ & Subsets & Statin & Follow & Results \\
\hline 1 & MIRACAL & 3088 & $\begin{array}{c}\text { ACS } \\
\text { (NSTEMI) }\end{array}$ & $\begin{array}{l}\text { Atorvastatin } \\
\quad(80 \mathrm{mg})\end{array}$ & $\begin{array}{c}16 \\
\text { weeks }\end{array}$ & $\begin{array}{l}16 \% \mathrm{RR} \text { in composite } \\
\text { end point of death } \mathrm{MI} \text { and } \\
\operatorname{TVR}(\mathrm{p}=0.48)\end{array}$ \\
\hline 2 & $\begin{array}{l}\text { PROVE-IT } \\
\text { TIMI } 22\end{array}$ & 4162 & $\begin{array}{c}\text { ACS } \\
\text { (NSTEMI \& } \\
\text { STEMI) }\end{array}$ & $\begin{array}{l}\text { Atorvastatin } \\
\quad(80 \mathrm{mg})\end{array}$ & 2 years & $\begin{array}{l}16 \% \mathrm{RR} \text { in all } \\
\text { cause mortality }\end{array}$ \\
\hline 3 & A to $\mathrm{Z}$ & 4497 & $\begin{array}{c}\text { ACS } \\
(\mathrm{NSTEM})\end{array}$ & $\begin{array}{c}\text { Simvastatin } \\
(40 \text { mg BD) }\end{array}$ & 2 years & $\begin{array}{l}\text { Non significant trend } \\
(p=0.14) \text { in favour of high dose } \\
\text { simvastatin }\end{array}$ \\
\hline 4 & ARMYDA-ACS & 191 & $\begin{array}{c}\text { PCI } \\
\text { to STEMI }\end{array}$ & $\begin{array}{l}\text { Atorvastatin } \\
\quad(80 \mathrm{mg})\end{array}$ & 30 days & $\begin{array}{l}\text { Significant reduction of } \\
\text { composite end point of death } \\
\text { MI and } \operatorname{TVR}(\mathrm{p}=0.01)\end{array}$ \\
\hline
\end{tabular}

\section{From clinical trials to guideline} recommendation:

The results of MIRACAL and CHAMP studies among other studies, prompted the National Cholesterol Education Program to revise the approach taken to initiating lipid lowering medication. The NCEP - ATP III guidelines recommended in-hospital initiation of lipid lowering medications in conjunction with diet and exercise counseling as the standard of care. The PRVE ITTIMI 22 trial neither has nor greatly strengthened the case for intensive LDL-C lowering immediately following an ACS. ${ }^{28}$ A revision ${ }^{29}$ to the NCEP-ATP III guidelines states that statin therapy should be considered for all patients who have experienced an acute coronary event irrespective of their baseline LDL-C level. The American College of Cardiology/American Heart Association now recommend with class I indication that statin therapy be initiated son after an ACS If LDL-C levels are $>100 \mathrm{mg} / \mathrm{dL}$ and that statin therapy be considered( class IIA indication) in patients with ACS if LDL-C levels are $<100 \mathrm{mg} / \mathrm{dL}$ or unknown. ${ }^{30}$
Very high risk patients- Patients with established cardiovascular disease plus

1. Multiple risk factors (especially Diabetes).

2. Sever and poorly controlled risk factors (especially continued cigarette smoking)

3. Multiple risk factors of the metabolic syndrome (especially triglycerides $\geq 200 \mathrm{mg} / \mathrm{dL}$ plus non HDL-C $\geq 130 \mathrm{mg} / \mathrm{dL}$ with HDL-C $<40 \mathrm{mg} / \mathrm{dL}$. plus

4. Patients with acute coronary syndromes.

High risk patients- Patients with CHD without high risk characteristics as indicated above, or patients with CHD risk equivalent

Is LDL below $70 \mathrm{mg} \%$ safe or unphysiological?

There should be any serious concern about dropping LDL cholesterol levels too low, because cholesterol delivery to peripheral tissue such as adrenal gland occurs mainly via HDL. At birth our $\mathrm{LDL}$ is $40 \mathrm{mg} / \mathrm{dL}$. In utero the $\mathrm{LDL}$ is $25 \mathrm{mg} / \mathrm{dL}$. Normal LDL-C range is $50-70 \mathrm{mg} / \mathrm{dL}$ for healthy

Table-II

Drug therapy consideration and goals of therapy for secondary prevention ${ }^{29}$

\begin{tabular}{lcc}
\hline & \multicolumn{2}{c}{ LDL consideration } \\
Risk category & Levels at which to consider drug therapy & Primary goal of therapy \\
\hline Very high risk $^{\text {a }}$ & $\geq 70 \mathrm{mg} / \mathrm{dL}$ & $<70 \mathrm{mg} / \mathrm{dL}$ \\
High risk100mg/dL ${ }^{\mathrm{b}}$ & $\geq 100 \mathrm{mg} / \mathrm{dL}$ & $<100 \mathrm{mg} / \mathrm{dL}$ \\
\hline
\end{tabular}


human neonates, native hunter gatherers, free living primates and other wild mammals who do not develop atherosclerosis. In PROVE IT-TIMI 22 sub study $91 \%$ of patients had $\mathrm{LDL}<100 \mathrm{mg} / \mathrm{dL}$ at the end of 4 month and there was no significant differences in safety parameters including muscle, liver, retinal changes, intracranial haemorrhage or death. ${ }^{31}$ groups with LDL $<40 \mathrm{mg} / \mathrm{dL}$ and $40-$ $60 \mathrm{mg} / \mathrm{dL}$ had fewer major CV events. Therefore there is no reason to fear vary low LDL. In fact, aggressive LDL lowering results in incremental benefits without additional safety concerns.

\section{Statin safety:}

No increase in non cardiovascular mortality has been observed in patients randomized to active treatment in any of the large statin. Furthermore, none of these trials suggests lower boundary below which LDL-C lowering is ineffective or dangerous. In PROVE-IT trials the subgroups that achieved LDL-C, $40 \mathrm{mg} / \mathrm{dL}$ and 40-60 mg/dL had fewer major cardiac events with no significant difference in safety outcomes. ${ }^{32}$ Most patients tolerate statins with few side effect. Occasional patient will have a mild rise in liver transaminases, but this change is not believed to be an indication of hepatotoxicity. Statin induced myopathy, defined as a serum creatinine kinase level of more than 10 times the upper limit of normal, has been observed in 0.1 to 0.5 percent of patients treated with statins during randomized controlled trials. ${ }^{33}$ In a series of 45 cases of statin induced myopathy the average duration of statin therapy prior to symptom onset was 6 months, and muscle pain resolve after an average of 2 months. ${ }^{34}$ In that series, 4-10 patients tolerated another statin without recurrent symptoms. Some patients receiving statins develop muscle symptoms but have normal serum creatinine kinase levels. Biopsy confirmed myopathy has been documented in such patient; ${ }^{35}$ consequently, normal creatinine kinase levels do not rule out statin induced myopathy in patients with muscle symptoms. The frequency of this condition in unknown.

Rhabdomyolysis characterize by muscle weakness myoglobinuria, and renal failure, is a complication of statin therapy. In a series of patients with statin associates myopathy, 13\% develop rhabdomyolysis requiring hospitalization. ${ }^{34}$ Analysis from a database of more than 250,000 patients from managed care health plans found the average incidence of rhabdomyolysis for monotherapy with atorvastatin, pravastatin or simvastatin was 0.44 per 10,000 person-years. ${ }^{34}$

\section{Conclusion:}

Available evidence from clinical trials suggests that, compared with placebo or moderate dose statin treatment, high dose intensive statin therapy significantly reduce incidents cardiovascular events. This benefit which was observe in both individuals with stable CAD as well as those with recent acute coronary event is likely to been independent of concomitant treatment and, in post ACS scenario, it may be attributable at least in part to the non lipid lowering pleotropic effects of statins, such as decreased inflammation.. Although, to date, trials have not demonstrated a major increase in the incidence of serious adverse events among subjects treated with high dose regimens warring withdrawal of such treatment, subclinical elevation of liver enzymes was particularly common in intensively treated group. In addition, prior to initiating high dose statin therapy in individual patients, it is important to consider the fact that most studies demonstrated a decrease in nonfatal cardiovascular outcomes and nonfatal end points, owing to lack of adequate power. Thus a balanced approach would be to recommend high dose intensive statin treatment to all subjects with an ACS and to reserve such treatment to subjects with stable CAD who have not met the LDL-C target less than $70 \mathrm{mg} / \mathrm{dL}$ on moderate dose statin therapy.

\section{Future perspective:}

Results of future clinical trials on intensive statin therapy could provide useful additional information if they are able to demonstrate a clear benefit for all major cardiovascular outcomes, especially hard end points, such as fatal MI. This can be achieved by conducting studies adequately powered for each clinical end points. Formal cost benefit analyses could be conducted to establish with certainty, the financial implication embarking on a high dose statin therapy.

\section{Conflict of Interest- None.}

\section{References:}

1. Genest J, Libby P, Lipoprotein disorders and cardiovascular disease. In: Libby P, Bonow RO, Mann, DL, Zipes DP (eds) Braunwald's heart Disease: A text book of cardiovascular disease. $8^{\text {th }}$ edn. Saunders, Philadelphia. 2008. : Pp 1071-1092.

2. Ridker PM, JUPITER Study Group: Rosuvastatin in the primary prevention of cardiovascular disease among 
patients with low levels of low-density lipoprotein cholesterol and elevated high sensitive C Reactive protein: Rationale and design of the JUPITER trial. Circultaion 2003;108:2292.

3. Davignon J: Beneficial cardiovasculkar pleotrophic effect of ststins. Circulation 2004; 109(Suppl): III 39.

4. Libby P, Aikawa: Stabilazitaion of arherosclerotic plaque: New mwchanism and clinical targets. Nat med 2002; 8: 1257.

5. Granger CB,Weaver WD. Reducing cardiac events after acute coronary syndromes. Rev Cardiovasc Med 2004; 5 : S39-S46.

6. Shin J, Edelberg JE, Hong MK. Vulnerable atherosclerotic plaque: clinical implications. Curr Vasc Pharmacol 2003; 1: 183-204.

7. Moustapha A, Anderson HV, Contemporary view of the acute coronary syndromes. J Invasive Cardiol 2003 15: 71-79.

8. American Heart Association. Heart disease and stroke statistics: 2003 update. Dallas TX: American Heart Association 2002.

9. Law M, Wald N, Rudnicka A. Quantifying effect of statins on low density lipoprotein cholesterol, ischaemic heart disease, and stroke: systemic review and metaanalysis. BMJ 2003: 326: 1423-1429.

10. Pepine CJ. Optimizing lipid management in patients with acute coronary syndromes. Am J Cardiol 2003: 30B-35B.

11. Massberg S, Schulz C, Gawaz M. Role of plateletsin the Pathophysiology of acute coronary syndrome. Semin Vasc Med 2003; 3: 148 -162.

12. Strong JP, Malcom GT, McMahan CA, et al. Prevalence and extent of atherosclerosis in adolescents and young adults: Implications for prevention from the pathological determinants of atherosclerosis in Youth Study. JAMA 1999; 281: 727-735.

13. Gallo R, Badimon JJ, Fuster V, Althouth Pathology of coronary ischaemic events: clinical implications. Adv Inter Med 1998; 43: 203-232.

14. Libby P. Current concepts of the pathogenesis of the acute coronary syndromes. Circulation 2001; 342: 101114.

15. Yeghiazarians Y, Braunstein JB, Askari A et al. Unstable angina pectoris. N Eng Med J 2000; 342: 101114.

16. Waters DD. Early pharmacological intervention and plaque stability in acute coronary syndrome. $\mathrm{Am} \mathrm{J}$ Cadriol 2001; 88: 30K-36K.

17. Reiner Z. Should we begin with statin therapy in acute coronary syndrome? Acta Med Croatica 2004; 58:147150.

18. Nissen SE, High dose statins in acute coronary syndrome: not just lipid levels. JAMA 2004; 292: 13651367.

19. Ridker PM. High sensitivity C-reactive protein: potential adjunct for global risk assessment in the primary prevention of cardiovascular diseases. Circulation 2001; 103: $1813-1818$.

20. Ridker PM, Cannon CP, Morrow D, et al. C reactive protein levels and outcomes afterb sstatin therapy. $N$ Eng J Med 2005; 352: 20-28.
21. Aronow HD, Topol EJ, Roe MT, et al> Effect of lipid lowering therapy on early mortality after acute coronary syndromes: an observational study. Lancet 2001; 357:1063-1068.

22. Scwartz GG, Olsson AG, Ezekowitz MD, et al. Myocardial Ischaemia Reduction with Aggressive Cholesterol Lowering (MIRACAL) Study Investigators. Effect of atorvastatin on early recurrent ischaemic events in acute coronary syndromes: The MIRACAL study; a randomized controlled trial. JAMA 2001; 285:1711-1718.

23. Canon CP, Braunwald E, McCabe $\mathrm{CH}$, et al. Intensive versus moderate lipid lowering with statins after ACS. $N$ Eng J Med 2004; 350:1495-1504.

24. de Lemos JA, Blazing MA, Wiviott SD, et al. Early intensive vs. a delayed conservative simvastatin strategy in patients with acute coronary syndromes: phase Z of the A to Z trial JAMA 2004; 292:1307-1316.

25. Fonarow GC, Gawlinski A. Rationale and design of the Cardiac Hospitalization Atherosclerosis management Programme at the University of California, Los Angeles. Am J Cardiol 2000;85:10A-17A.

26. Study of the effectiveness of Additional Reductions of Cholesterol and Homocystein. 9 Nov.2008. www.ctsu.ox.ac.uk.

27. Patti G, Pasceri V, Colona G et al. Atorvastatin pretreatment improves outcomes in patients with acute coronary syndromes undergoing early coronary intervention: results of ARMYDA-ACS (Atorvastatin for reduction of Myocardial Damage during Angioplasty- Acute Coronary Syndrome) randomized trial. J am Coll Cardiol 2007; 49: 1272-1278.

28. Cannon CP, Braunwald E, McCabe $\mathrm{CH}$, et al. Intensive versus moderate lipid lowering with statins after acute coronary syndromes. N Eng J Med 2004; 350: 14951504.

29. Grund SM, Cleeman JI, Merz CN, et al. Implications of recent clinical trials for the National Cholesterol Education Program Adult Treatment Panel III guidelines. Circulation 2004; 110: 227-1239.

30. Heart Protection Study Collaborative Group. MRC/ BHF Heart Protection Study of cholesterol lowering with simvastatin in 20536 high risk individuals: a randomized placebo controlled trial. Lancet 2002; 360: 7-22.

31. Ray JG, Gomg Y, Sykora K, et al. statin use and survival outcomes in elderly patients with heart failure. Arch Intern Med 2005; 165:62-67.

32. Wiviott SD, Cannon CP, Morrow DA, et al. Can low density lipoprotein be too low? The safety and efficacy of achieving very low low-density density lipoprotein with intensive statin therapy: a PROVE-IT TIMI 22 sub study. J Am Cardiol 2005; 46:1411-1416.

33. Graham DJ, Staffa JA, Shatin D, et al. Incidence of hospitalized rhabdomyolysis in patients treated with lipid lowering drugs. JAMA 2004; 292:2585-2590.

34. Hansen KE, Hidelbrand JP, Ferguson EE, et al. Outcomes in 45 patients with statin-associated myopathy. Arch Intern Med 2005; 165:2671-2676.

35. Philips PS, Hass RS, Bannykh S, et al. Statin associated myopathy with normal creatinine kinase levels. Ann Itern Med 2002,137581-585. 